\title{
0 PROCESSO DE CONSENTIMENTO LIVRE E ESCLARECIDO EM PESQUISA: UMA NOVA ABORDAGEM
}

José Roberto Goldim*, Carolina da Fonte Pithan, Juliana Ghisleni de Oliveira, Márcia Mocelin Raymundo

Trabalho realizado no Hospital de Clínicas de Porto Alegre, Rio Grande do Sul, RS.

RESUMO - OBjetivos. 0 processo de consentimento livre e esclarecido tem por objetivo permitir que a pessoa que está sendo convidada a participar de um projeto de pesquisa compreenda os procedimentos, riscos, desconfortos, benefícios e direitos envolvidos, visando permitir uma decisão autônoma. 0 objetivo do presente estudo foi avaliar a possibilidade de fornecer informações de forma coletiva na obtenção do consentimento livre e esclarecido.

Métodos. Em um projeto de pesquisa da área da Ginecologia foi utilizada a transmissão coletiva de informações, através de uma palestra, para a obtenção do consentimento livre e esclarecido. Foram entrevistadas 45 participantes deste projeto, verificando a recordação dos procedimentos, riscos e benefícios.

Resultados. Todas as participantes (100\%) recordaram os procedimentos, $54 \%$ delas recordaram os riscos e $96 \%$ os benefícios prováveis. Comparando os presentes resultados com os obtidos em um estudo similar utilizando o processo de consentimento livre e esclarecido convencional (transmissão individual de informações), verificou-se que os sujeitos de pesquisa recordaram mais informações no atual estudo.

CoNCLUsöEs. Estes resultados indicam a possibilidade de que as informaçōes necessárias ao consentimento livre e esclarecido possam ser dadas de forma coletiva, nos projetos em que esta alternativa seja adequada.

Unitermos: Consentimento livre e esclarecido. Pesquisa em seres humanos.

\section{INTRODUÇÃO}

A pesquisa em seres humanos tem 0 compromisso de resguardar a integridade de todos os envolvidos. Este resguardo envolve questões sobre a preservação da privacidade, a minimização de riscos e desconfortos, a busca de benefícios, a não discriminação e a proteção de grupos de pessoas vulneráveis. Duas estratégias têm sido utilizadas no intuito de proteger os indivíduos estudados: a utilização de consentimento livre e esclarecido e a avaliação por Comitês de Ética em Pesquisa'.

A avaliação prévia dos projetos feita por um Comitê de Ética em Pesquisa visa assegurar que os aspectos éticos e metodológicos estejam adequados. Este Comitê, devido a sua independência e representatividade acadêmica e social, deve garantir que as pesquisas tenham um aval institucional, além da responsabilidade já assegurada pelo pesquisador ${ }^{2}$.

*Correspondência:

Grupo de Pesquisa e Pós-Graduação

Rua Ramiro Barcellos, 2350 - sala 2227

90035-003 - Porto Alegre - RS

Tel. (52) 3316-8000 - R. 8304
Desde 1993, as questões referentes ao processo de consentimento livre e esclarecido vêm sendo pesquisadas pelo Núcleo Interdisciplinar de Bioética, vinculado ao Grupo de Pesquisa e Pós-Graduação do Hospital de Clínicas de Porto Alegre (GPPG/HCPA)

$O$ processo de consentimento livre e esclarecido tem por objetivo permitir que a pessoa que está sendo convidada a participar de um projeto de pesquisa compreenda os procedimentos, riscos, desconfortos, benefícios e direitos envolvidos, visando permitir uma decisão autônoma. A obtenção de consentimento livre e esclarecido é um dever moral do pesquisador, é a manifestação do respeito às pessoas envolvidas no projeto ${ }^{5}$. O Termo de Consentimento Livre e Esclarecido documenta a autorização do sujeito da pesquisa e permite que as informações básicas possam ser mantidas para leitura posterior ${ }^{6}$.

Diferentemente da relação estabelecida emassistência, que envolve apenas o médicoe seu paciente, na pesquisa a relação se estabelece entre o pesquisador e, no mínimo, uma amostra de sujeitos participantes da pesquisa. Habitualmente, a obtenção do consentimento livre e esclarecido é realizada entre o profissional e o participante, o que pode diminuir a voluntariedade na participação e gerar constrangimentos, pois muitas vezes o vínculo assistencial pode tolher a liberdade de escolha.

Em um estudo anterior, realizado com 56 participantes de seis diferentes projetos de pesquisa, foiverificado que o item que mais se associava à recordação de procedimentos, riscos e benefícios foi o fornecimento de informações prévias à leitura do Termo de Consentimento Livre e Esclarecido?.

Algumas estratégias têm sido utilizadas para melhorar a qualidade do processo de obtenção do consentimento livre e esclarecido. Murphy et al. alteraram a forma do Termo de Consentimento Livree Esclarecido incluindo ilustrações ealterando a dificuldade de leitura do texto em si. As alterações propiciaram uma maior compreensão das informações prestadas ${ }^{8}$.

Com a finalidade de estudar uma alternativa ao processo de consentimento livree esclarecido usual foi realizado um estudo com o objetivo de avaliar a possibilidade de fornecer informações, de forma coletiva, sobre o projeto de pesquisa, previamente à obtenção do Termo de Consentimento Livre e Esclarecido. Isto foi avaliado em termos da recordação sobre procedimentos, riscos e benefícios. 


\section{Métodos}

A primeira etapa do presente estudo foi selecionar um projeto de pesquisa, aprovado pelo Comitê de Ética em Pesquisa do Hospital de Clínicas de Porto Alegre, onde a transmissão de informações, visando à obtenção do consentimento livre e esclarecido sobre o mesmo pudesse ser realizada de forma coletiva. Dentre os inúmeros projetos aprovados para serem realizados no HCPA, e que ainda não haviam iniciado, foi selecionado um projeto daárea da Ginecologia, que consistia em um levantamento epidemiológico sobre a prevalência e a incidência da infecção pelo papilomavírus humano $(\mathrm{HPV})$ em adolescentes e mulheres adultas. O pesquisador responsável do projeto concordou com a proposta e colaborou na execução do presente estudo.

As informações foram fornecidas às potenciais participantes e as seus responsáveis legais através de uma palestra na qual foram apresentados os procedimentos, riscos e benefícios da pesquisa para todas as participantes. Esta apresentação teve apoio de material audiovisual. Foi possibilitado que as pessoas presentes que não tivessem interesse em participar, após terem recebido as informações, se retirassem do local de realização antes da obtenção do consentimento propriamente dito. As que permaneceram no local foi apresentado o Termo de Consentimento Livre e Esclarecido, assim como foram dirimidas eventuais dúvidas. Cabe salientar que o Termo de Consentimento Livre e Esclarecido foi lido e assinado de forma individual, constituindo-se em um único documento por participante.

Para permitir o levantamento de dados no presente estudo, foi elaborada uma lista dos procedimentos, riscos e benefícios que constavam no projeto de pesquisa e no Termo de Consentimento Livre e Esclarecido. Esta lista foi elaborada com a finalidade de verificar o grau de recordação das informações fornecidas.

Foram entrevistadas 45 mulheres das 250 que aceitaram participar do projeto de pesquisa, imediatamente após a obtenção do Termo de Consentimento Livre e Esclarecido. Todas as participantes selecionadas assistiram a mesma palestra em um mesmo grupo. Os critérios de inclusão foram ter mais de 18 anos e ter assinado o Termo de Consentimento Livre e Esclarecido. Esta amostra

Tabela I - Grau de recordação de procedimentos, riscos e benefícios em duas diferentes formas de fornecimento de informações, individual ou coletiva, visando a obtenção do consentimento livre e esclarecido

$\begin{array}{ccc}\begin{array}{c}\text { Informações } \\ \text { recordadas }\end{array} & \begin{array}{c}\text { Transmissão } \\ \text { individual } \\ (\mathrm{n}=56)\end{array} & \begin{array}{c}\text { Transmissão } \\ \text { coletiva } \\ (\mathrm{n}=45)\end{array} \\ \text { Procedimentos } & 47,5 \% & 100 \% \\ \text { Riscos } & 22 \% & 54 \% \\ \text { Benefícios } & 47,5 \% & 96 \%\end{array}$

aleatória simples representou $18 \%$ das participantes. O cálculo do tamanho da amostra foi realizado com base nos dados de estudo anterior neste mesmo tema ${ }^{7}$.

Por ser um estudo que envolvia o próprio processo de obtenção do Consentimento Livre e Esclarecido e pelo fato de não agregar riscos, pois foi feita apenas uma entrevista com a aplicação de um instrumento de avaliação não-invasivo à privacidade, foi solicitada a dispensa da utilização de um Termo de Consentimento Livre e Esclarecido por escrito, de acordo com o item IV.3.f da Resolução 196/966 , ao Comitê de Ética em Pesquisa do HCPA. O consentimento livre e esclarecido dos indivíduos foi solicitado verbalmente.

\section{Resultados e Discussão}

Foram incluídas no presente estudo 45 mulheres participantes do projeto de pesquisa selecionado. Ainclusão destas participantes foi feita de maneira aleatória. As participantes foram entrevistadas, tendo sido verificado 0 grau de recordação das informações sobre procedimentos, riscos e benefícios que haviam sido apresentadas quando da obtenção do consentimento livre e esclarecido. $O$ processo de consentimento livre e esclarecido incluiuas informações dadas durante a palestra explicativa e no esclarecimento de eventuais dúvidas por parte das participantes. Aentrevista foi realizada imediatamente após a obtenção do consentimento livre e esclarecido.

A totalidade das participantes do presente estudo (100\%) recordou os procedimentos aos quais seriam submetidas. Do mesmo modo, pôde-se verificar que $54 \%$ delas recordaram os riscose $96 \%$ os benefícios prováveis vinculados ao projeto de pesquisa.

De 1995 a 1999, foi realizado um estudo similar com uma amostra de 56 participantes de seis diferentes projetos de pesquisa do HCPA.
Este estudo avaliou, do mesmo modo, a recordação dos procedimentos, riscos e benefícios pelos participantes desses projetos. Nesta ocasião, todas as informações foram prestadas a cada um dos indivíduos em particular. Os participantes recordaram $47,5 \%$ dos procedimentos, $22,0 \%$ dos riscos e $47,5 \%$ dos benefícios ${ }^{7}$.

Godwin et al. realizaram um estudo semeIhante em mulheres submetidas a mamoplastia. As pacientes recordaram apenas $25 \%$ das informações que foram fornecidas no processo de consentimento livre e esclarecido. Grande parte do grupo estudado (74\%) sugeriu que deveriam ser utilizados recursos visuais no processo?.

A baixa recordação das informações pode ser creditada, pelo menos em parte, à ansiedade associada ao ato de consentir ou não coma participação em um projeto de pesquisa (Tabela I). Não existem estudos específicos que expliquem porque a recordação dos riscos no processo de consentimento livre e esclarecido é inferior a dos procedimentos e benefícios que foi verificada em ambos estudos realizados. Uma possível explicação pode estar associada à forma de lidar com os conflitos e incertezas proposto por Hofstede ${ }^{10,11}$.

A leitura do texto do Termo de Consentimento Livree Esclarecido foi considerada acessível por $91 \%$ das participantes. No outro estudo, envolvendo seis projetos de pesquisa, menos de $50 \%$ dos participantes acharam os Termos de Consentimento Livree Esclarecido acessíveis ${ }^{7}$. O resultado obtido em relação à leitura do Termo de Consentimento pode ser atribuído, pelo menos em parte, às informações prévias recebidas durantea palestra por todas as participantes. Vale salientar que o Termo de Consentimento utilizado no projeto de pesquisa avaliado foi elaborado pelos próprios pesquisadores, não tendo sido alterado para ter uma estrutura de texto evocabulário mais acessíveis. 


\section{Conclusão}

A transmissão coletiva de informações no processo de consentimento livre e esclarecido auxilia na adequada compreensão do projeto de pesquisa, pois permite mais tempo e o uso de outros recursos, tais como meios áudiovisuais, habitualmente não utilizados na sua obtenção. O indivíduo adequadamente informado está em melhores condições de exercer livremente a sua escolha entre participar ou não de uma pesquisa. Estas características melhoram a qualidade global do consentimento obtido.

\footnotetext{
SUMMARY

INFORMED CONSENT IN RESEARCH: A NEW APPROACH

INTRODUCTION. The Informed Consent allows the person invited to participate of a reserch project to understand the procedures, risks, discomforts, benefits and rights involved; determining an autonomic decision. We want to verify in the informed consent process the adequacy of given information to research subjects in a collective way.

METHODS. A research project was chosen from the gynecologic area. The collective obtainment consisted of an oral presentation. After that, The Consent form was shown. Fortyfive patients were interwied, immediately after the consent obtainment.
}

RESULTS. Remembrance capacity of information about procedures, risks and benefits explained were verified. All participants (100\%) remembered the procedures, $54 \%$ of them remembered the risks and $96 \%$ the probable benefits. Comparing these data with others from a similar study using conventional informed consent process (individual information transmission) the subjects remember more information in the present study.

CONCLUSION. These results evidence the possibility to inform collectively the research subjects when getting the Informed Consent in those projects where this choice is available. [Rev Assoc Med Bras 2003; 49(4): 372-4]

KEYWORDS: Informed consent. Clinical trails.

\section{REFERÊNCIAS}

I. U.S. President's Commission for the Study of Ethical Problems in Medicine and Biomedical and Behavioral Research. Deciding to forego life-sustaining treatment: a report on the ethical, medical, and legal issues in treatment decisions. Washington, D.C.: U.S. Government Printing Office; 1983.

2. Goldim JR, Francisconi CF. Os comitês de ética hospitalar. Rev Med ATM 1995; I5(I):327-34.

3. Matte U, Goldim JR. Pesquisa em saúde: aspectos éticos e metodológicos envolvidos na avaliação de projetos de pesquisa. Rev HCPA \& Fac Med Univ Fed Rio Gd do Sull 995; 15(2): 135.
4. Raymundo MM, Matte U, Goldim JR. Consentimento Informado e avaliação de projetos de pesquisa no período de 1996 a 1997. Rev HCPA \& Fac Med Univ Fed Rio Gd do Sul 1998; I8(supl):30-I.

5. Clotet J. O consentimento informado nos Comitês de Ética em pesquisa e na prática médica: conceituação, origens e atualidade. Bioética 1995; 3(I):5I-9.

6. Brasil. Conselho Nacional de Saúde. Resolução 196/96 sobre pesquisas envolvendo seres humanos. Diário Oficial da União, Brasília, I6 out. 1996. p.21082-2 1085.

7. Goldim JR. O consentimento informado e a adequação de seu uso na pesquisa em seres humanos [tese]. Porto Alegre: Universidade Federal do Rio Grande do Sul; 1999.

8. Murphy DA, O'Keefe ZH, Kaufman AH. Improving comprehension and recall of information for an HIV vaccine trial among women at risk for HIV: reading level simplification and inclusion of pictures to illustrate key concepts. AIDS Educ Prev 1999; I I (5):389-99.

9. Godwin Y. Do they listen? A review of information retained by patients following consent for reduction mammoplasty. Br JPlast Surg 2000; 53(2): | $2 \mid-5$.

10. Hosfstede G. Culture and organizations. New York: McGraw Hill, 1997. p.23-47, 109-38.

II. Clotet J, Goldim JR, Francisconi CF. Consentimento Informado e a sua prática na assistência e pesquisa no Brasil. Porto Alegre: EDIPUCRS, 2000. p.II9.

Artigo recebido: 05/08/2002

Aceito para publicação: 29/05/2003 